WSU-HEP-2001

April 6, 2020

\title{
Model Independent Extraction of the Proton Charge Radius from PRad data
}

\author{
GIL PAZ \\ Department of Physics and Astronomy \\ Wayne State University, Detroit, Michigan 48201, USA
}

\begin{abstract}
The proton radius puzzle has motivated several new experiments that aim to extract the proton charge radius and resolve the puzzle. Recently PRad, a new electron-proton scattering experiment at Jefferson Lab, reported a proton charge radius of $0.831 \pm$ $0.007_{\text {statistical }} \pm 0.012_{\text {systematic }}$. The value was obtained by using a rational function model for the proton electric form factor. We perform a model-independent extraction using z-expansion of the proton charge radius from PRad data. We find that the modelindependent statistical error is more than $50 \%$ larger compared to the statistical error reported by PRad.
\end{abstract}




\section{Introduction}

The proton is a composite particle. One way to define its size is by the proton charge radius, $r_{E}^{p}$. It is related to the slope of the proton electric form factor, $G_{E}^{p}$, at $q^{2}=0$, see (2) below. Since $G_{E}^{p}$ is a non-perturbative function of $q^{2}$, its slope must be extracted from data. The most direct way to measure $r_{E}^{p}$ is by extracting $G_{E}^{p}$ from lepton-proton scattering and finding its slope at $q^{2}=0$. An indirect way is by using atomic spectroscopy.

Thus we have four different methods to extract $r_{E}^{p}$ from data: $e-p$ scattering, $\mu-p$ scattering, $e-p$ spectroscopy, and $\mu-p$ spectroscopy. A fifth method, Lattice QCD, should become competitive in the future, see, e.g., [1]. While $e-p$ scattering and spectroscopy extractions were available for a long time, $\mu-p$ spectroscopy only became available in 2010 from the work of the CREMA collaboration $[2,3]$. Results from $\mu-p$ scattering are expected in the near future from the MUSE collaboration [4]. Ideally, all methods should give consistent results. Surprisingly, in 2010, $\mu-p$ spectroscopy gave a value, $0.84184(67) \mathrm{fm}$, that was considerably smaller than the CODATA value, 0.8768(69) fm [5]. This difference is referred to as the "proton radius puzzle". For a recent review, see [6].

The puzzle has motivated new theoretical and experimental work. Three new $e-p$ spectroscopy measurements were published recently. Two agree with the smaller value $[7,8]$, and one [9] with the larger value. Two new $e-p$ scattering experiments, ISR and PRad, have published their results and more experiments are planned [10]. ISR found 0.81(8) fm [11], and more recently [12] $0.87(4) \mathrm{fm}$, that cannot distinguish between the two values. PRad found [13] $0.831 \pm 0.007_{\text {statistical }} \pm 0.012_{\text {systematic }} \mathrm{fm}$, which favors the smaller value.

A main issue in extracting $r_{E}^{p}$ from scattering data is the unknown functional form of $G_{E}^{p}$. Recent extractions have used: dipole [14], polynomial [15, 16], continued fraction [15], modified $z$ expansion [17], or more complicated forms [18]. For pre-2010 extractions see [19]. Different assumed functional forms can lead to different radii and uncertainties from the same data. An alternative approach is the so-called $z$ expansion that only uses the known analytic structure of $G_{E}^{p}$. The $z$ expansion is the default method for meson form factors. It was first applied to baryon form factors in [20]. Extractions of $r_{E}^{p}$ using $z$ expansion favor the larger value $[20,21]$.

The default functional form for $G_{E}^{p}$ used by PRad is a rational function called "Rational $(1,1)$ ", see (5) below. Apart from the overall normalization (that does not affect the slope) it depends on two parameters. In [20] it was shown that a fit with a small number of parameters can underestimate the errors. In figure S15 of the supplementary material of the PRad paper [13], the "Rational (1,1)" fit and "2 nd order $z$-tran." give similar radii with similar uncertainty, but " 3 rd order $z$-tran." has twice the uncertainty. As was shown in [20], adding higher powers of $z$ without bounding the coefficients will cause the uncertainty to grow without bound. On the other hand, if we bound the coefficients, we obtain an extraction of $r_{E}^{p}$ that is independent of the number of the parameters we fit [20]. Since the form factor must have the correct analytic structure and therefore can be expanded as a power series in $z$, we obtain an extraction of $r_{E}^{p}$ that is independent of the exact unknown functional form of the form factor.

The goal of this paper is to perform such a model-independent analysis to the published PRad data and to see how it affects the errors on the extracted $r_{E}^{p}$. For simplicity, we use 
the values of $G_{E}^{p}$ reported by PRad in [22] and use only the statistical errors ${ }^{1}$. The rest of the paper is organized as follows. In section 2 we briefly review the relevant form factor parameterization and the $z$ expansion. In section 3 we repeat the fits performed by PRad to its data and reproduce their results. In section 4 we perform a model-independent $z$-expansion fit to the PRad data and extract $r_{E}^{p}$. We present our conclusions in section 5.

\section{Form factor parameterization and the $z$ expansion}

The one-photon probe of the proton gives rise to two form factors, $F_{1}^{p}$ and $F_{2}^{p}$,

$$
\left\langle P\left(p^{\prime}\right)\left|J_{\mu}^{\mathrm{em}}\right| P(p)\right\rangle=\bar{u}\left(p^{\prime}\right)\left[\gamma_{\mu} F_{1}^{p}\left(q^{2}\right)+\frac{i \sigma_{\mu \nu}}{2 m_{p}} F_{2}^{p}\left(q^{2}\right) q^{\nu}\right] u(p),
$$

where $q^{2}=\left(p^{\prime}-p\right)^{2} \equiv t \equiv-Q^{2}$. The electric and magnetic form factors are defined as [23] $G_{E}^{p}\left(q^{2}\right)=F_{1}^{p}\left(q^{2}\right)+q^{2} F_{2}^{p}\left(q^{2}\right) / 4 m_{p}^{2}$ and $G_{M}^{p}\left(q^{2}\right)=F_{1}^{p}\left(q^{2}\right)+F_{2}^{p}\left(q^{2}\right)$. The proton charge radius squared is defined via the slope of $G_{E}^{p}\left(q^{2}\right)$ at $q^{2}=0$ :

$$
\left\langle r^{2}\right\rangle_{E}^{p}=\left.\frac{6}{G_{E}^{p}(0)} \frac{d}{d q^{2}} G_{E}^{p}\left(q^{2}\right)\right|_{q^{2}=0} .
$$

The proton charge radius is given by $r_{E}^{p} \equiv \sqrt{\left\langle r^{2}\right\rangle_{E}^{p}}$. In [13] $r_{E}^{p}$ is denoted by $r_{p}$.

$G_{E}^{p}\left(q^{2}\right)$ is analytic in the complex $q^{2}$ plane outside a cut that starts at the two-pion threshold $q^{2}=4 m_{\pi}^{2}$. The domain of analyticity can be mapped onto the unit $|z|<1$ circle via the transformation

$$
z\left(t, t_{\mathrm{cut}}, t_{0}\right)=\frac{\sqrt{t_{\mathrm{cut}}-t}-\sqrt{t_{\mathrm{cut}}-t_{0}}}{\sqrt{t_{\mathrm{cut}}-t}+\sqrt{t_{\mathrm{cut}}-t_{0}}},
$$

where $t_{\text {cut }}=4 m_{\pi}^{2}$ and $t_{0}$ determines the location of $z=0$. In the following we use $t_{0}=0$. In the $|z|<1$ unit circle $G_{E}^{p}$ is analytic and can be expanded as a power series:

$$
G_{E}^{p}\left(q^{2}\right)=\sum_{k=0}^{\infty} a_{k} z\left(q^{2}\right)^{k}
$$

The choice $t_{0}=0$ implies that $r_{E}^{p}$ depends only on $a_{1}$.

Plotting the data as function of $z$ can be very instructive. For example, when only a slope can be constrained, the $z$-dependent data will appear linear, while the $Q^{2}$-dependent data can appear to have curvature. See, e.g., figure 3 in [24] for a mesonic form factor and figure 2 in [25] for a baryonic form factor. In figure 1 we plot $G_{E}^{p}$ from the full PRad data set (described in the next section) as a function of $Q^{2}$ (left) and $z$ (right). We can see a certain amount of curvature in the plot of $G_{E}^{p}$ as a function of $z$. We will explore this further in section 4.3.

The default fit function used by PRad is

$$
f\left(Q^{2}\right)=n G_{E}^{p}\left(Q^{2}\right)=n \frac{1+p_{1} Q^{2}}{1+p_{2} Q^{2}} .
$$

\footnotetext{
${ }^{1}$ Determining the systematic error for the charge radius is much more involved and described in the supplementary material of the PRad paper [13].
} 

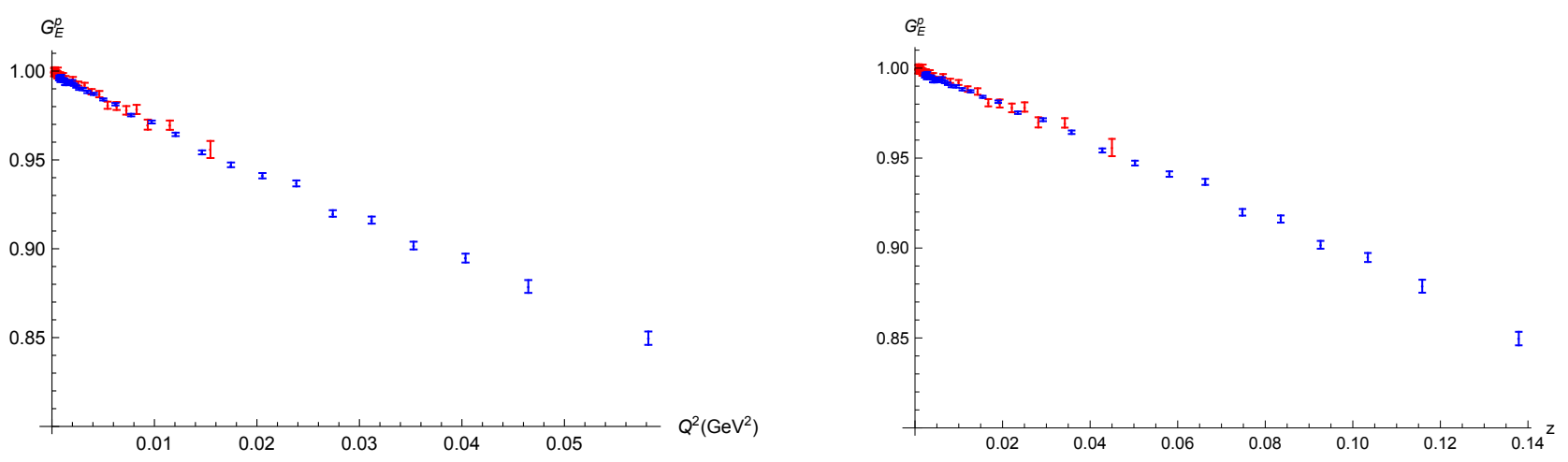

Figure 1: A comparison of $G_{E}^{p}$ from the PRad data set as a function of $Q^{2}$ (left) and $z$ (right). The $1.1 \mathrm{GeV}(2.2 \mathrm{GeV})$ data set is in red (blue), the same color scheme used in [13].

In [13] it is refereed to as "Rational $(1,1)$ ". This function can be written as a sum of pole and a constant:

$$
f\left(Q^{2}\right)=n \frac{1+p_{1} Q^{2}}{1+p_{2} Q^{2}}=n \frac{1-p_{1} t}{1-p_{2} t}=\frac{n\left(p_{1}-p_{2}\right) / p_{2}^{2}}{t-1 / p_{2}}+\frac{n p_{1}}{p_{2}} .
$$

Provided that $1 / p_{2}>4 m_{\pi}^{2}$, this function's singularity lies above the two-pion threshold. In order to have the correct analytic structure, we must have $1 / p_{2}>4 m_{\pi}^{2}$. We will check this requirement against PRad data in section 3.1.

Assuming $1 / p_{2}>4 m_{\pi}^{2}$, the Rational $(1,1)$ function can be expressed as a power series in $z$. The coefficients $a_{k}$ depend on its imaginary part. Since this is a sum of pole and a constant, the imaginary part is a delta function. Using the expressions in [20] we find

$$
\begin{aligned}
a_{0} & =f(0) \stackrel{\text { Rational }}{=}(1,1) \\
a_{k \geq 1} & =\frac{2}{\pi} \int_{t_{\text {cut }}}^{\infty} \frac{d t}{t-t_{0}} \sqrt{\frac{t_{\text {cut }}-t_{0}}{t-t_{\text {cut }}}} \operatorname{Im} f(t) \sin [k \theta(t)] \stackrel{\text { Rational }(1,1)}{=} \\
& =\frac{2 n\left(p_{1}-p_{2}\right)}{p_{2}} \sqrt{\frac{4 m_{\pi}^{2}}{p_{2}^{-1}-4 m_{\pi}^{2}}} \sin \left[k \cos ^{-1}\left(1-8 p_{2} m_{\pi}^{2}\right)\right] .
\end{aligned}
$$

We will compare these expressions to a z-expansion fit to the PRad data in section 4.3.

\section{$3 \quad$ PRad extractions of the proton charge radius}

Before improving on the $r_{E}^{p}$ extraction from the PRad data, we should be able to reproduce its published results. We use the information in [13] and its supplementary material. We use the PRad data release [22] from December 10, 2019. The "raw" values of $G_{E}^{p}\left(Q^{2}\right)$ can be obtained from the "1.1GeV_table.txt" and "2.2GeV_table.txt" files, where they are listed under "f(Q2)". The two files correspond to the 1.1-GeV and 2.2-GeV electron beams data of the PRad experiment. 
We repeat many of the fits reported in [13] and its supplementary material. We focus on the default PRad Rational $(1,1)$ fit and the fits involving the $z$ expansion. We use the $\chi^{2}$ function

$$
\chi_{\text {PRad }}^{2}=\sum_{i=1}^{N} \frac{\left(G_{E, \text { exp. }}^{p, i}-G_{E, \text { theo. }}^{p, i}\right)^{2}}{\left(\delta G_{E \text {,exp. }}^{p, i}\right)^{2}},
$$

and minimize it for a given theoretical expression of $G_{E}^{p}$. We use only the statistical errors in $\delta G_{E, \exp }^{p, i}$. The proton charge radius is calculated via (2). The uncertainty is found by the $\delta \chi^{2}=1$ range. In reproducing the PRad fits we follow its practice and include a normalization factor for the data as a multiplicative factor in $G_{E, \text { theo. }}^{p, i}$ This normalization factor is also determined from the fit.

\subsection{Default PRad fit}

The default expression for $G_{E}^{p}$ used by PRad is the Rational $(1,1)$ function given in (5). The Rational $(1,1)$ is fitted to both the $1.1 \mathrm{GeV}$ and $2.2 \mathrm{GeV}$ with different overall normalization factors called $n_{1}$ and $n_{2}$, but with the same $p_{1}$ and $p_{2}$.

From our fit we find $n_{1}=1.0002 \pm 0.0002_{\text {statistical }}, n_{2}=0.9983 \pm 0.0002_{\text {statistical }}$, and $r_{E}^{p}=0.831 \pm 0.007_{\text {statistical }} \mathrm{fm}$. The reduced $\chi^{2}$ is 1.3. These are also the results in [13].

As a further check, we find that the fit values of $p_{1}$ and $p_{2}$ are $p_{1}=-0.0715 \mathrm{GeV}^{-2}$ and $p_{2}=2.88 \mathrm{GeV}^{-2}$. Up to the first three significant figures, these are the values reported in "readme.pdf" in [22]. Including the uncertainties on these parameters, we find $p_{1}=-0.07_{-0.54}^{+0.56}$ $\mathrm{GeV}^{-2}$ and $p_{2}=2.88_{-0.59}^{+0.61} \mathrm{GeV}^{-2}$. Within the one standard deviation range we have $1 / p_{2}>$ $4 m_{\pi}^{2} \approx 0.0784 \mathrm{GeV}^{2}$. Thus the fit result is consistent with the analytic structure of $G_{E}^{p}$.

\subsection{Other PRad fits}

In the supplementary material of [13] the results of other fits to the PRad data are shown, but only in figures. Still, the approximate value of $r_{E}^{p}$ and its statistical uncertainty can be inferred from the figures.

PRad performed fits to its entire data set using a second order and third order polynomial in $z$. These correspond to truncating the series in (4) at $z^{2}$ and $z^{3}$, respectively. For example, equation (2) of the supplementary material is $f\left(Q^{2}\right)=n G_{E}^{p}\left(Q^{2}\right)=n\left(1+p_{1} z+p_{2} z^{2}\right)$. Using these expressions with different normalizations for the $1.1 \mathrm{GeV}$ and $2.2 \mathrm{GeV}$ data, our fit to the PRad data gives $r_{E}^{p}=0.830 \pm 0.008_{\text {statistical }}$ fm for the second order polynomial in $z$ and $r_{E}^{p}=0.825 \pm 0.015_{\text {statistical }} \mathrm{fm}$ for the third order polynomial in $z$. These results agree with the values and statistical uncertainty in figure S15 of the supplementary material of [13]. Notice also that the uncertainty is doubled when changing from a second to a third order polynomial. We will address this problem below.

PRad also performed fits using Rational $(1,1)$ to parts of the data set. These are listed in figure S16(a) of the supplementary material of [13]. Following PRad, we fitted the 1.1 $\mathrm{GeV}$ data, $2.2 \mathrm{GeV}$ data, $Q^{2}<0.016 \mathrm{GeV}^{2}$ data, and $Q^{2}>0.002 \mathrm{GeV}^{2}$ data. We find $r_{E}^{p}=0.845_{-0.039}^{+0.041} \mathrm{fm}$ for the $1.1 \mathrm{GeV}$ data only, $r_{E}^{p}=0.829_{-0.008}^{+0.008} \mathrm{fm}$ for the $2.2 \mathrm{GeV}$ data only, 

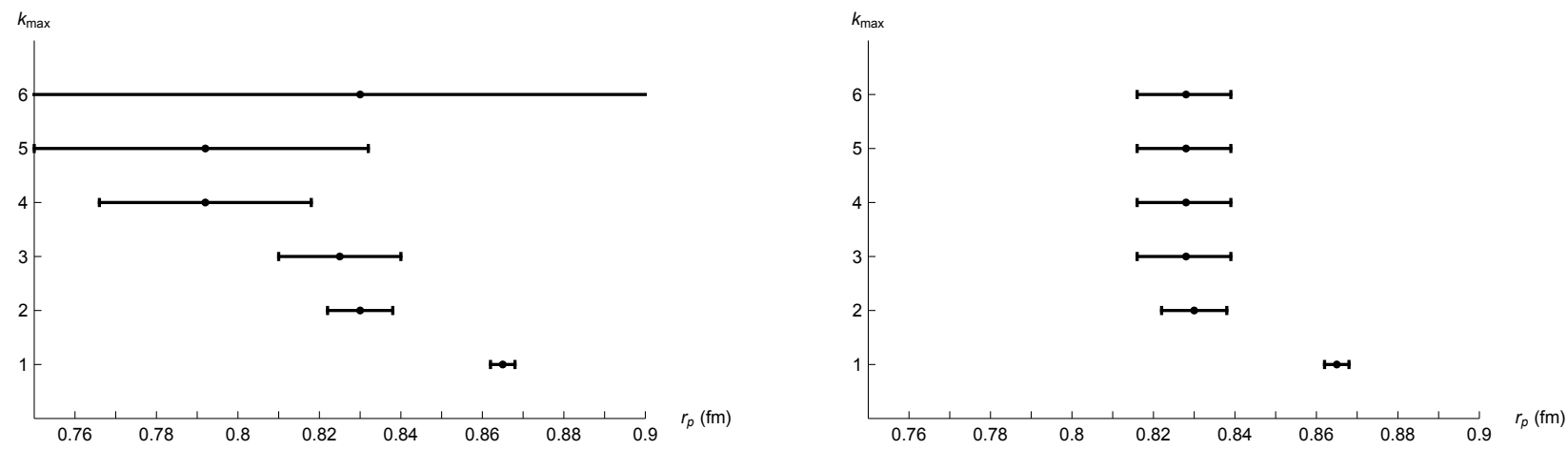

Figure 2: A comparison of the extracted $r_{E}^{p}$ as a function of the number of fitted $p_{k}$ parameters from $z$-expansion fits to the PRad data set. The range on the $x$-axis is the same range used in figure S15 of the supplementary material of [13]. Left: a fit without a bound on the coefficients $p_{k}$. Starting at $k_{\max }=5$ the uncertainty on $r_{E}^{p}$ exceeds the range $0.75-0.9 \mathrm{fm}$. Right: a fit with a bound of 5 on the coefficients $p_{k}$.

$r_{E}^{p}=0.799_{-0.017}^{+0.018} \mathrm{fm}$ for the $Q^{2}<0.016 \mathrm{GeV}^{2}$ data, and $r_{E}^{p}=0.841_{-0.011}^{+0.011} \mathrm{fm}$ for the $Q^{2}>0.002$ $\mathrm{GeV}^{2}$ data. All uncertainties are statistical. These results agree with figure S16(a).

Finally, PRad considered a fit of a second order polynomial in $z$ to the $2.2 \mathrm{GeV}$ data only. Performing such a fit we find $r_{E}^{p}=0.829 \pm 0.009_{\text {statistical }} \mathrm{fm}$. These results agree with figure S16(b) of the supplementary material of [13].

In conclusion, we reproduced the values of $r_{E}^{p}$ reported by PRad from the PRad data. We now investigate if and how these results change when we use a model-independent extraction.

\subsection{The need for a bound on the coefficients}

Truncating the z-expansion series, as was done in the PRad fits, might underestimate the uncertainty of $r_{E}^{p}$. On the other hand, simply increasing the number of fitted parameters can overestimate the uncertainty. As shown in [20], one needs to bound the coefficients.

To illustrate that, we perform a fit to the PRad data of the form $f\left(Q^{2}\right)=n G_{E}^{p}\left(Q^{2}\right)=$ $n\left(1+p_{1} z+p_{2} z^{2}+\cdots+p_{k_{\max }} z^{k_{\max }}\right)$. As in the PRad fits we use different normalization factors $n_{1}$ and $n_{2}$ for the $1.1 \mathrm{GeV}$ and the $2.2 \mathrm{GeV}$ data, but the same $p_{k}$ for both data sets. We consider two cases, no bound on $p_{k}$ and a bound $\left|p_{k}\right|<5$. We implement the bound as in [21] by adding $\chi_{\text {Bound }}^{2}=\sum_{k=0}^{k_{\max }} p_{k}^{2} / 5^{2}$ to $(9)$.

The results of the two fits are shown in figure 2 as a function of the number of fitted $p_{k}$ parameters. As expected [20], the uncertainty on the extracted value of $r_{E}^{p}$ grows without bound for the unbounded fit, while for the bounded fit it stabilizes on $r_{E}^{p}=0.828_{-0.012}^{+0.011} \mathrm{fm}$. 


\section{Model independent extraction of the proton charge radius}

Below we perform a model-independent z-expansion fit to PRad data, that includes a bound on the coefficients. We consider a fit to the whole PRad data set as well as the $1.1 \mathrm{GeV}$ and $2.2 \mathrm{Gev}$ data subsets. We also explore the effects of the bound on the coefficients, the $Q^{2}$ dependence of the extracted $r_{E}^{p}$, and the possible extraction of $a_{k}$ parameters beyond $a_{1}$.

\subsection{Model-independent $z$-expansion $r_{E}^{p}$ extraction from the entire PRad data}

We extract $r_{E}^{p}$ from the PRad data by using the $\chi^{2}$ function

$$
\chi_{z}^{2}=\sum_{i=1}^{N} \frac{\left(\eta_{i} G_{E, \text { exp. }}^{p, i}-G_{E, \text { theo. }}^{p, i}\right)^{2}}{\left(\delta G_{E, \text { exp. }}^{p, i}\right)^{2}}
$$

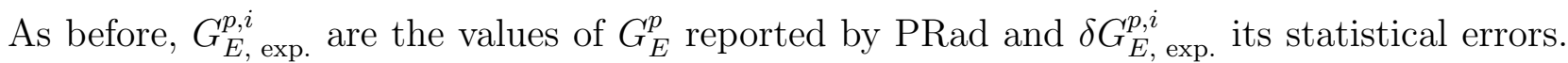
$G_{E, \text { theo. }}^{p, i}$ is given in (4) where the series is truncated at $k_{\max }$. The normalization factor are $\eta_{i}=\eta_{1}$ if $i$ is part of the $1.1 \mathrm{GeV}$ data set, and $\eta_{i}=\eta_{2}$ if $i$ is part of the $2.2 \mathrm{GeV}$ data set. Thus we allow for a normalization factor for each data set, but unlike PRad fits, we do not include it in $G_{E \text {, theo. }}^{p, i}$ Therefore this $\chi^{2}$ function differs from the one used in section 3.3. Since we include a normalization factor, we fix $G_{E}^{p}(0)=1$ which implies $a_{0}=1$ in the fits.

In order to bound the coefficients we add to $\chi_{z}^{2}$, as in [21], $\chi_{\text {Bound }}^{2}$ defined as

$$
\chi_{\text {Bound }}^{2}=\sum_{k=0}^{k_{\max }} \frac{a_{k}^{2}}{B^{2}},
$$

where $B$ is a pure number. Our default value is $B=5$, but we check our results also for $B=10$. This choice of bounds was discussed in detail and implemented in the literate, see $[20,25,21]$ for the nucleon electromagnetic form factors and $[26,27,28]$ for the nucleon axial form factor.

Fitting the entire PRad data with $B=5$, we find that the extracted proton charge radius is $r_{E}^{p}=0.828_{-0.012}^{+0.011} \mathrm{fm}$. Changing the bound to $B=10$ we find $r_{E}^{p}=0.827_{-0.014}^{+0.013} \mathrm{fm}$. These are almost identical one-standard deviation ranges. Compared to the default PRad fit of Rational $(1,1), r_{E}^{p, \text { rational }}=0.831 \pm 0.007 \mathrm{fm}$, the central values are almost the same, but the uncertainty is more than $50 \%$ larger for the $z$-expansion fit. The extracted $r_{E}^{p}$ stabilizes for $k_{\max }=3$. It does not change as we increase $k_{\max }$ above 3 . We checked this with fits up to $k_{\max }=10$.

As another check, we consider fits without adding $\chi_{\text {Bound }}^{2}$ and by using explicit bounds of $\left|a_{k}\right| \leq 5$ and $\left|a_{k}\right| \leq 10$ as in [20,25]. We find $r_{E}^{p}=0.824_{-0.012}^{+0.015}$ fm for $\left|a_{k}\right| \leq 5$ and $r_{E}^{p}=0.824_{-0.015}^{+0.015} \mathrm{fm}$ for $\left|a_{k}\right| \leq 10$. These are very close to the results above that used $\chi_{\text {Bound }}^{2}$. 


\subsection{Model-independent $z$-expansion $r_{E}^{p}$ extraction from parts of the PRad data}

The Rational $(1,1)$ fits to the $1.1 \mathrm{GeV}$ and $2.2 \mathrm{GeV}$ parts of the PRad data give values of $r_{E}^{p}$ that are within the one-standard deviation range of each other, but the uncertainty on the former is five times as large. It is instructive to see what are the results for $z$-expansion fit. We use the same fit $\chi^{2}$ function, namely the sum of $\chi_{z}^{2}$ and $\chi_{\text {Bound }}^{2}$.

Using only the $1.1 \mathrm{GeV}$ data set we find $r_{E}^{p}=0.847_{-0.037}^{+0.035} \mathrm{fm}$ for $B=5$ and $r_{E}^{p}=0.846_{-0.041}^{+0.039}$ $\mathrm{fm}$ for $B=10$. These are almost identical to the Rational $(1,1)$ fit result of $r_{E}^{p \text {,rational }}=$ $0.845_{-0.039}^{+0.041} \mathrm{fm}$. Using only the $2.2 \mathrm{GeV}$ data set we find $r_{E}^{p}=0.826_{-0.013}^{+0.012} \mathrm{fm}$ for $B=5$ and $r_{E}^{p}=0.823_{-0.015}^{+0.015} \mathrm{fm}$ for $B=10$. These are consistent with the Rational $(1,1)$ fit result of $r_{E}^{p \text {,rational }}=0.829_{-0.008}^{+0.008} \mathrm{fm}$, but the uncertainty is more than $50 \%$ larger for the $z$-expansion fit.

Another question we study is the effect of a cut on $Q^{2}$. We consider this question for the 1.1 GeV data alone, the $2.2 \mathrm{GeV}$ data alone, and the combined $1.1 \mathrm{GeV}$ and $2.2 \mathrm{GeV}$ data. We perform fits to $r_{E}^{p}$ for data with $Q^{2}$ smaller than $Q_{\text {cut }}^{2}$. The lowest $Q_{\text {cut }}^{2}$ is determined by the requirement that the slope of the form factor is positive. If $Q^{2}$ is too small, we do not have enough data for a meaningful extraction of $r_{E}^{p}$. Thus $Q_{\text {cut }}^{2} \in[0.0007,0.0155] \mathrm{GeV}^{2}$ for the 1.1 $\mathrm{GeV}$ data set, $Q_{\text {cut }}^{2} \in[0.001,0.059] \mathrm{GeV}^{2}$ for the $2.2 \mathrm{GeV}$ data set, and $Q_{\text {cut }}^{2} \in[0.0007,0.059]$ $\mathrm{GeV}^{2}$ for the combined $1.1 \mathrm{GeV}$ and $2.2 \mathrm{GeV}$ data sets. All fits are performed with $B=5$.

The results of the extractions are shown in figure 3 . In all three plots we see a convergence to a value as $Q_{\text {cut }}^{2}$ is increased. In the $2.2 \mathrm{GeV}$ data set plot and the combined $1.1 \mathrm{GeV}$ and 2.2 $\mathrm{GeV}$ data sets we see also a "peak" at about $Q_{\text {cut }}^{2}=0.0014 \mathrm{GeV}^{2}$. But overall the extraction is independent of the cut on $Q^{2}$, for large enough $Q_{\text {cut }}^{2}$.

\subsection{Model-independent $z$-expansion fit to the entire PRad data}

The charge radius is only a one-parameter characterization of the data. We can try and extract more coefficients in (4). To do that we use (9) and add to it a modified version of (10) where we omit the term $a_{i}^{2} / B^{2}$ in the sum in (10) when constraining the $a_{i}$ coefficient.

We perform such a fit to the full PRad data set (both 1.1 and $2.2 \mathrm{GeV}$ ) using $B=5$. The fit stabilizes quickly for $k_{\max }=i+3$. We find that $a_{1}=-0.921 \pm 0.026, a_{2}=-1.2 \pm 0.6$, and $a_{3}=2.2 \pm 5.7$. Using $B=10$ gives very similar results. This implies that beyond a slope $\left(a_{1}\right)$, only a curvature $\left(a_{2}\right)$ can be obtained from the PRad data ${ }^{2}$.

To compare these results graphically to the PRad data, we perform a fit with $B=5$ to the full PRad data set without bounding $a_{1}$ and $a_{2}$, i.e., we omit the terms $a_{1}^{2} / B^{2}$ and $a_{2}^{2} / B^{2}$ in the sum in (10). The fit stabilizes quickly with increasing $k_{\max }$. We find the values above for $a_{1}$ and $a_{2}$ and a covariance of -0.0137 between them. The variance of the data normalization factors $\eta_{1,2}$ is negligible as well as their covariance with $a_{1}$ or $a_{2}$. The resulting fit and uncertainty [29] is shown in figure 4 together with the PRad data from figure 1.

\footnotetext{
${ }^{2}$ We can compare these values to the values predicted by the Rational $(1,1)$ fit from section 3.1 . Using $p_{1}=-0.07_{-0.54}^{+0.56} \mathrm{GeV}^{-2}$ and $p_{2}=2.88_{-0.59}^{+0.61} \mathrm{GeV}^{-2}$, equation (7) gives $a_{1}^{\text {Rational }(1,1)}=-0.93 \pm 0.26$ and $a_{2}^{\text {Rational }(1,1)}=-1.02 \pm 0.19$. These agree with the values we obtained from the $z$-expansion fit.
} 

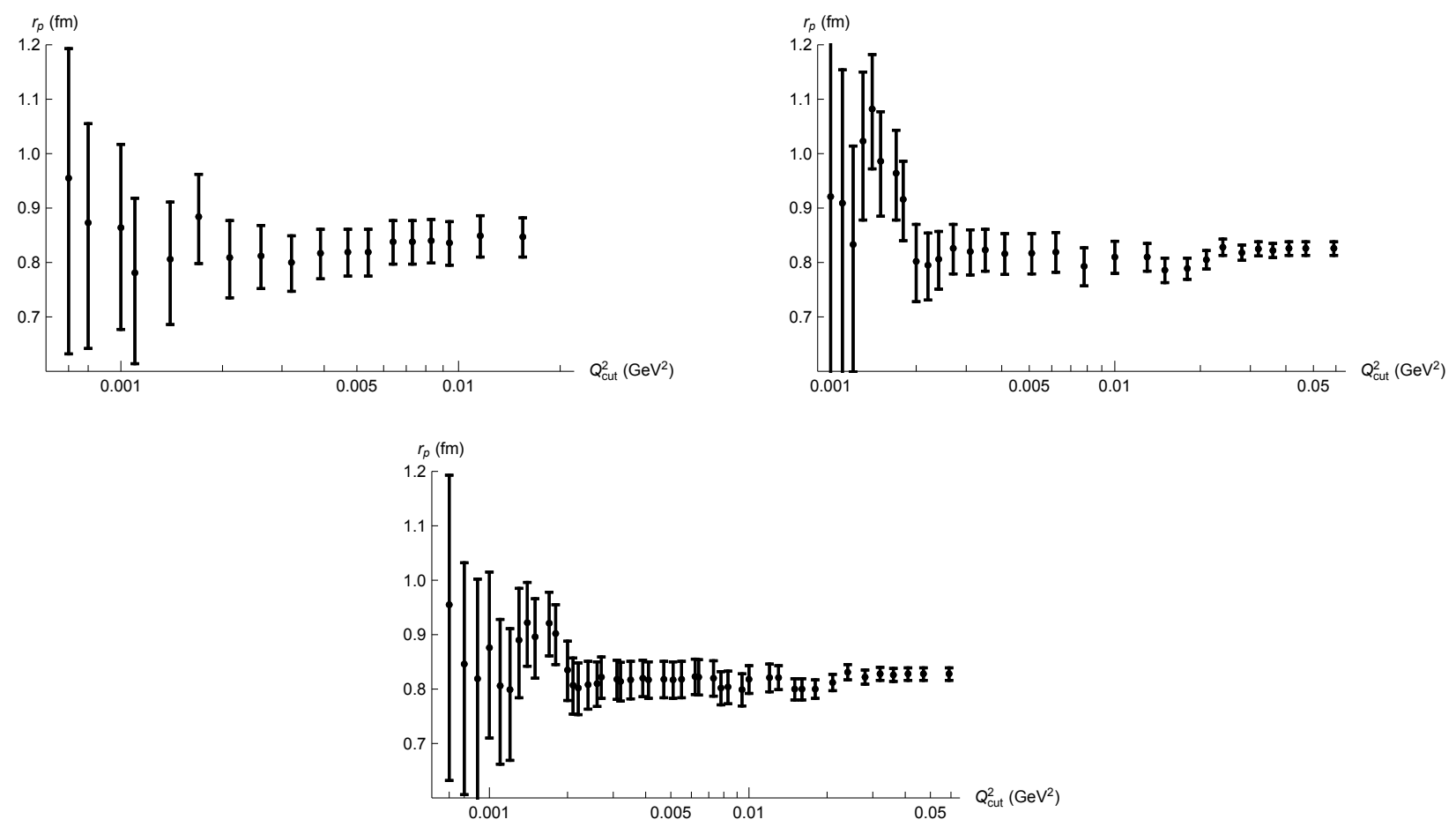

Figure 3: Extracted $r_{E}^{p}$ as a function of $Q_{\text {cut }}^{2}$. Top left: $1.1 \mathrm{GeV}$ data set. Top right: $2.2 \mathrm{GeV}$ data set. Bottom center: 1.1 and $2.2 \mathrm{GeV}$ data sets.

\section{Conclusions}

The proton radius puzzle has motivated new theoretical and experimental work. Among them is PRad, a new electron-proton scattering experiment at Jefferson Lab. PRad reached the lowest $Q^{2}$ in $e-p$ scattering: $2.1 \times 10^{-4} \mathrm{GeV}^{2}$, an order of magnitude lower than previously achieved at A1 Mainz [30,31]. The small $Q^{2}$ should allow to reduce extrapolation errors in extracting the proton charge radius.

PRad has extracted a radius of $0.831 \pm 0.007_{\text {statistical }} \pm 0.012_{\text {systematic }}$ fm by using a Rational $(1,1)$ fit function for $G_{E}^{p}$. Instead of relying on a specific model for $G_{E}^{p}$, one can use a modelindependent approach via the $z$ expansion. In this paper we have examined how the statistical error reported by PRad changes when using such a model-independent approach.

In section 3 we repeated many of the fits performed by PRad to its data. These include its default Rational $(1,1)$ fit to the entire PRad data, a second and third order polynomial in $z$ fit to the entire PRad data, Rational $(1,1)$ fit to parts of its data set, and a second order polynomial in $z$ fit to its $2.2 \mathrm{GeV}$ data. These agree with [13], its supplementary material, and information from the PRad data release [22] from December 10, 2019.

We also compared the extractions of $r_{E}^{p}$ when higher polynomials in $z$ are considered, with and without bounding the coefficients of the polynomial in $z$. The results appear in figure 2 . As expected [20], we find that the extracted proton charge radius grows without bound for the unbounded fit, while for the bounded fit it stabilizes very quickly to a value independent 

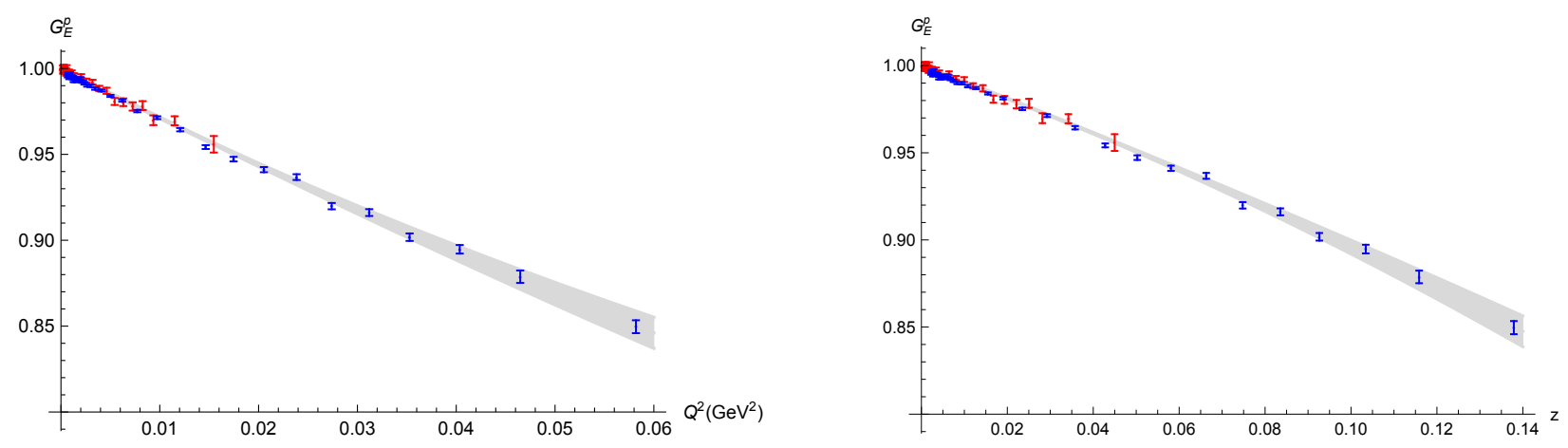

Figure 4: A model-independent z-expansion fit to $G_{E}^{p}$ from the entire PRad data set (gray band) together with values of $G_{E}^{p}$ from the PRad data set as a function of $Q^{2}$ (left) and $z$ (right). The $1.1 \mathrm{GeV}(2.2 \mathrm{GeV})$ data set is in red (blue), the same color scheme used in [13].

of the degree of the $z$ polynomial.

In section 4 we performed a model-independent $z$-expansion fit to PRad data. The bounding of the coefficients is implemented by adding a term to $\chi^{2}$ [21], see (10). From a fit to the entire PRad data set we find $r_{E}^{p}=0.828_{-0.012}^{+0.011} \mathrm{fm}$. Compared to the default PRad fit, $r_{E}^{p \text {,rational }}=0.831 \pm 0.007 \mathrm{fm}$, the central values are almost the same, but the uncertainty is more than $50 \%$ larger for the z-expansion fit. This implies that PRad's default fit underestimates the statistical error by using the Rational $(1,1)$ function.

We also performed a model-independent z-expansion fit to parts of the PRad data. We fitted the $1.1 \mathrm{GeV}$ and $2.2 \mathrm{GeV}$ parts of the PRad data separately. For the $1.1 \mathrm{GeV}$ data (that contains the smaller $Q^{2}$ data) we find that the model independent extraction is almost identical to the Rational $(1,1)$ fit. The error bar of this extraction is too large to distinguish between the two values of the proton charge radius. For the $2.2 \mathrm{GeV}$ data the model independent extraction uncertainty is $50 \%$ larger than the Rational $(1,1)$ fit. We considered also the effects of a $Q^{2}$ cut on the data, $Q^{2}<Q_{\text {cut }}^{2}$. The results are shown in figure 3 for the $1.1 \mathrm{GeV}$ data, the $2.2 \mathrm{GeV}$ data, and the entire PRad data. Overall the extraction is independent of the cut on $Q^{2}$, for a large enough $Q_{\text {cut }}^{2}$.

Going beyond $r_{E}^{p}$, we fitted more parameters in the $z$ expansion to the PRad data. The results are described in section 4.3 and figure 4 . We find that beyond the slope, equivalent to $r_{E}^{p}$, only a curvature can be obtained from the PRad data.

Before concluding, let us briefly review recent papers that also analyzed the PRad data. In [32] PRad data was analyzed to investigate their consistency with $r_{E}^{p}$ from muonic hydrogen and theoretical predictions for the coefficients of $Q^{4}$ and $Q^{6}$ terms in the $Q^{2}$ expansion of $G_{E}^{p}$. Using a rational function to incorporate these inputs, the author of [32] found very good agreement with the PRad data. In [33] a fit using the DI $\chi$ EFT model to the PRad and A1 Mainz data [30, 31] was performed. The authors of [33] found the same value of $r_{E}^{p}$ within uncertainties as their fit to A1 Mainz data alone. Finally, very recently [34] appeared that compared fits using the z-expansion to non-PRad scattering data and PRad data. The authors of [34] remark that their z-expansion fit to PRad data, taking the PRad errors at face value, 
results in a significantly larger uncertainty for $r_{E}^{p}$ compared to the Rational $(1,1)$ PRad fit.

In summary, using model-independent methods we find that the statistical uncertainty on the proton charge radius from the PRad data is more than $50 \%$ larger than the one quoted by PRad in [13]. The systematic error is obtained by a much more involved process that is described in the supplementary material of the PRad paper [13]. It is likely that the systematic error will also increase when using model-independent methods. It is needed for a full model-independent extraction of the proton charge radius from the PRad data.

\section{Acknowledgements}

We thank Haiyan Gao, Claude Pruneau, and Weizhi Xiong for useful discussions. This work was supported by the U.S. Department of Energy grant DE-SC0007983 and by a Career Development Chair award from Wayne State University.

\section{References}

[1] C. Alexandrou, K. Hadjiyiannakou, G. Koutsou, K. Ottnad and M. Petschlies, [arXiv:2002.06984 [hep-lat]].

[2] R. Pohl et al., Nature 466, 213 (2010).

[3] A. Antognini et al., Science 339, 417 (2013).

[4] R. Gilman et al. [MUSE Collaboration], arXiv:1709.09753 [physics.ins-det].

[5] P. J. Mohr, B. N. Taylor and D. B. Newell, Rev. Mod. Phys. 80, 633 (2008) [arXiv:0801.0028 [physics.atom-ph]].

[6] G. Paz, eConf C1907293 (2019) [arXiv:1909.08108 [hep-ph]].

[7] A. Beyer et al., Science 358, 79 (2017).

[8] N. Bezginov et al., Science 365, 1007 (2019).

[9] H. Fleurbaey et al., Phys. Rev. Lett. 120, 183001 (2018) [arXiv:1801.08816 [physics.atom$\mathrm{ph}]$.

[10] See the talks at the 2018 MITP workshop "Precision Measurements and Fundamental Physics: The Proton Radius Puzzle and Beyond" https://indico.mitp.uni-mainz.de/event/132/timetable/\#all

[11] M. Mihovilovič et al., Phys. Lett. B 771, 194 (2017) [arXiv:1612.06707 [nucl-ex]].

[12] M. Mihovilovič, P. Achenbach, T. Beranek, J. Beričič, J. C. Bernauer, R. Böhm, D. Bosnar, M. Cardinali, L. Correa and L. Debenjak, et al. Eur. Phys. J. A 57, no.3, 107 (2021) [arXiv:1905.11182 [nucl-ex]].

[13] W. Xiong et al., Nature 575, no. 7781, 147 (2019). 
[14] D. W. Higinbotham, A. A. Kabir, V. Lin, D. Meekins, B. Norum and B. Sawatzky, Phys. Rev. C 93, 055207 (2016) [arXiv:1510.01293 [nucl-ex]].

[15] K. Griffioen, C. Carlson and S. Maddox, Phys. Rev. C 93, no. 6, 065207 (2016) [arXiv:1509.06676 [nucl-ex]].

[16] M. Horbatsch, E. A. Hessels and A. Pineda, Phys. Rev. C 95, 035203 (2017) [arXiv:1610.09760 [nucl-th]].

[17] M. Horbatsch and E. A. Hessels, Phys. Rev. C 93, 015204 (2016) [arXiv:1509.05644 [nucl-ex]].

[18] J. M. Alarcón, D. W. Higinbotham, C. Weiss and Z. Ye, Phys. Rev. C 99, 044303 (2019) [arXiv:1809.06373 [hep-ph]].

[19] K. Nakamura et al. [Particle Data Group], J. Phys. G 37, 075021 (2010).

[20] R. J. Hill and G. Paz, Phys. Rev. D 82, 113005 (2010) [arXiv:1008.4619 [hep-ph]].

[21] G. Lee, J. R. Arrington and R. J. Hill, Phys. Rev. D 92, no. 1, 013013 (2015) [arXiv:1505.01489 [hep-ph]].

[22] https://wiki.jlab.org/pcrewiki/index.php/PRad_Results

[23] F. J. Ernst, R. G. Sachs and K. C. Wali, Phys. Rev. 119, 1105 (1960).

[24] R. J. Hill, eConf C 060409, 027 (2006) [hep-ph/0606023].

[25] Z. Epstein, G. Paz and J. Roy, Phys. Rev. D 90, no.7, 074027 (2014) [arXiv:1407.5683 [hep-ph]].

[26] B. Bhattacharya, R. J. Hill and G. Paz, Phys. Rev. D 84, 073006 (2011) [arXiv:1108.0423 [hep-ph]].

[27] B. Bhattacharya, G. Paz and A. J. Tropiano, Phys. Rev. D 92, no.11, 113011 (2015) [arXiv:1510.05652 [hep-ph]].

[28] A. S. Meyer, M. Betancourt, R. Gran and R. J. Hill, Phys. Rev. D 93, no.11, 113015 (2016) [arXiv:1603.03048 [hep-ph]].

[29] C. A. Pruneau, "Data Analysis Techniques for Physical Scientists," (Cambridge University Press, Cambridge, 2017).

[30] J. Bernauer et al. [A1], Phys. Rev. Lett. 105, 242001 (2010) [arXiv:1007.5076 [nucl-ex]].

[31] J. Bernauer et al. [A1], Phys. Rev. C 90, no.1, 015206 (2014) [arXiv:1307.6227 [nucl-ex]].

[32] M. Horbatsch, Phys. Lett. B 804, 135373 (2020) [arXiv:1912.01735 [nucl-ex]].

[33] J. Alarcón, D. Higinbotham and C. Weiss, [arXiv:2002.05167 [hep-ph]]

[34] K. Borah, R. J. Hill, G. Lee and O. Tomalak, [arXiv:2003.13640 [hep-ph]]. 